International Journal of Pure and Applied Mathematics

Volume 98 No. 4 2015, 503-510

ISSN: 1311-8080 (printed version); ISSN: 1314-3395 (on-line version)

url: http://www.ijpam.eu

doi: http://dx.doi.org/10.12732/ijpam.v98i4.9

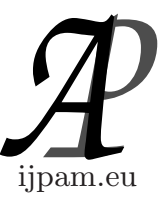

\title{
APPROXIMATE ANALYTICAL SOLUTION OF THE TIME FRACTIONAL WHITHAM-BROER-KAUP EQUATION USING THE HOMOTOPY ANALYSIS METHOD
}

\author{
Ali Kurt ${ }^{1}$, Orkun Tasbozan ${ }^{2} \S$ \\ ${ }^{1,2}$ Department of Mathematics \\ Faculty of Science and Art \\ Mustafa Kemal University \\ Hatay, 31000, TURKEY
}

\begin{abstract}
In this paper, the homotopy analysis method (HAM) is applied to the time fractional Whitham-Broer-Kaup equation to obtain its approximate analytical solutions. The HAM solution includes an auxiliary parameter $\hbar$ which provides a suitable way of adjusting and controlling the convergence region of solution series.
\end{abstract}

AMS Subject Classification: 35A25, 58B05

Key Words: homotopy analysis method, approximate analytical solution, the time fractional Whitham-Broer-Kaup equation

\section{Introduction}

When many physical events in various fields of physics and engineering are modelled, nonlinear ordinary or partial differential equations arise. Investigating and constructing exact and numerical solutions of these equations are of great importance in applied mathematics. Liao presented the $\operatorname{HAM}[1,2]$ which is a powerful tool for searching the approximate solutions of nonlinear evolution equations (NLEEs). Differently from perturbation techniques, the HAM is not

Received: October 5, 2014

(C) 2015 Academic Publications, Ltd.

$\S$ Correspondence author url: www.acadpubl.eu 
limited to any small physical parameters in the considered equation. Hence, the previous restrictions and limitations of perturbation techniques can be overcame by the HAM, so it enables us with a powerful tool to analyze strongly nonlinear problems[3]. Many authors have applied this method successfully to solve several nonlinear problems arising in science and engineering [1-8]. In this paper, we will apply the HAM to time fractional Whitham-Broer-Kaup equation.

\section{Application of the HAM}

We handle the time fractional Whitham-Broer-Kaup equation in the following form

$$
\begin{aligned}
& D_{t}^{\alpha} u+u u_{x}+H_{x}+q u_{x x}=0, \\
& D_{t}^{\alpha} H+(H u)_{x}+p u_{x x x}-q H_{x x}=0
\end{aligned}
$$

with initial conditions

$$
\begin{aligned}
& u(x, 0)=1-\tanh (x / 2), \\
& H(x, 0)=\frac{3}{4}\left(1-\tanh ^{2}(x / 2)\right)
\end{aligned}
$$

For all calculations, we take $p$ and $q$ as 3 and 1 , respectively in this paper. To investigate the series solution of Eqs. (1) with initial conditions (2), we choose the linear operators

$$
\mathcal{L}\left[\phi_{i}(x, t ; p)\right]=D_{t}^{\alpha} \phi_{i}(x, t ; p), \quad i=1,2,
$$

with the property

$$
\mathcal{L}\left[c_{i}\right]=0
$$

where $c_{i}^{\prime} \mathrm{s}(i=1,2)$ are constants. From Eq. (1), we now define nonlinear operators as

$$
\begin{aligned}
\mathcal{N}_{1}\left[\phi_{1}(x, t ; p), \phi_{2}(x, t ; p)\right] & =\frac{\partial^{\alpha} \phi_{1}(x, t ; p)}{\partial t^{\alpha}}+\phi_{1}(x, t ; p) \frac{\partial \phi_{1}(x, t ; p)}{\partial x} \\
& +\frac{\partial \phi_{2}(x, t ; p)}{\partial x}+\frac{\partial^{2} \phi_{1}(x, t ; p)}{\partial x^{2}}, \\
\mathcal{N}_{2}\left[\phi_{1}(x, t ; p), \phi_{2}(x, t ; p)\right] & =\frac{\partial^{\alpha} \phi_{2}(x, t ; p)}{\partial t^{\alpha}}+\frac{\partial \phi_{1}(x, t ; p) \phi_{2}(x, t ; p)}{\partial x} \\
& +3 \frac{\partial^{3} \phi_{1}(x, t ; p)}{\partial x^{3}}-\frac{\partial^{2} \phi_{2}(x, t ; p)}{\partial x^{2}} .
\end{aligned}
$$


Therefore, we set up the zero-order deformation equations as

$$
\begin{aligned}
(1-p) \mathcal{L}\left[\phi_{1}(x, t ; p)-u_{0}(x, t)\right] & =p \hbar \mathcal{N}_{1}\left[\phi_{1}(x, t ; p), \phi_{2}(x, t ; p)\right], \\
(1-p) \mathcal{L}\left[\phi_{2}(x, t ; p)-H_{0}(x, t)\right] & =p \hbar \mathcal{N}_{2}\left[\phi_{1}(x, t ; p), \phi_{2}(x, t ; p)\right] .
\end{aligned}
$$

Obviously, if we choose $p=0$ and $p=1$ then we obtain

$$
\phi_{1}(x, t ; 0)=u_{0}(x, t)=u(x, 0), \phi_{2}(x, t ; 0)=H_{0}(x, t)=H(x, 0)
$$

and

$$
\phi_{1}(x, t ; 1)=u(x, t), \phi_{2}(x, t ; 1)=H(x, t),
$$

respectively. Then, while the embedding parameter $p$ increases from 0 to 1 , the solutions $\phi_{1}(x, t ; p)$ and $\phi_{2}(x, t ; p)$ change from the initial values $u_{0}(x, t)$ and $H_{0}(x, t)$ to the solutions $u(x, t)$ and $H(x, t)$. By expanding $\phi_{1}(x, t ; p)$ and $\phi_{2}(x, t ; p)$ in Taylor series with respect to the embedding parameter $p$, we get

$$
\begin{aligned}
& \phi_{1}(x, t ; p)=u_{0}(x, t)+\sum_{m=1}^{\infty} u_{m}(x, t) p^{m}, \\
& \phi_{2}(x, t ; p)=H_{0}(x, t)+\sum_{m=1}^{\infty} H_{m}(x, t) p^{m}
\end{aligned}
$$

where

$$
\begin{aligned}
& u_{m}(x, t)=\left.\frac{1}{m !} \frac{\partial^{m} \phi_{1}(x, t ; p)}{\partial p^{m}}\right|_{p=0}, \\
& H_{m}(x, t)=\left.\frac{1}{m !} \frac{\partial^{m} \phi_{2}(x, t ; p)}{\partial p^{m}}\right|_{p=0} .
\end{aligned}
$$

If the auxiliary linear operator, the initial guess and the auxiliary parameter $\hbar$ are suitably chosen, the above series converges at $p=1$, and one has

$$
\begin{gathered}
u(x, t)=u_{0}(x, t)+\sum_{m=1}^{\infty} u_{m}(x, t), \\
H(x, t)=H_{0}(x, t)+\sum_{m=1}^{\infty} H_{m}(x, t),
\end{gathered}
$$

which must be one of the solutions of the original nonlinear equations, as proved by Liao $[2,5]$. By differentiating Eqs. (3) and (4) $m$ times with respect to the embedding parameter $p$, we obtain the $m$ th-order deformation equations

$$
\mathcal{L}\left[u_{m}(x, t)-\chi_{m} u_{m-1}(x, t)\right]=\hbar R_{1, m}\left(\mathbf{u}_{m-1}, \mathbf{H}_{m-1}\right),
$$




$$
\mathcal{L}\left[H_{m}(x, t)-\chi_{m} H_{m-1}(x, t)\right]=\hbar R_{2, m}\left(\mathbf{u}_{m-1}, \mathbf{H}_{m-1}\right)
$$

where

$$
\begin{aligned}
R_{1, m}\left(\mathbf{u}_{m-1}, \mathbf{H}_{m-1}\right) & =\frac{\partial^{\alpha} u_{m-1}(x, t)}{\partial t^{\alpha}}+\sum_{n=0}^{m-1} u_{n}(x, t) \frac{\partial u_{m-1-n}(x, t)}{\partial x} \\
& +\frac{\partial H_{m-1}(x, t)}{\partial x}+\frac{\partial^{2} u_{m-1}(x, t)}{\partial x^{2}}, \\
R_{2, m}\left(\mathbf{u}_{m-1}, \mathbf{H}_{m-1}\right) & =\frac{\partial^{\alpha} H_{m-1}(x, t)}{\partial t^{\alpha}}+\sum_{n=0}^{m-1} u_{n}(x, t) \frac{\partial H_{m-1-n}(x, t)}{\partial x} \\
& +\sum_{n=0}^{m-1} H_{n}(x, t) \frac{\partial u_{m-1-n}(x, t)}{\partial x}+3 \frac{\partial^{3} u_{m-1}(x, t)}{\partial x^{3}} \\
& -\frac{\partial^{2} H_{m-1}(x, t)}{\partial x^{2}} .
\end{aligned}
$$

The solutions of the $m$ th-order deformation Eqs. (9) and (10) for $m \geq 1$ leads to

$$
\begin{aligned}
u_{m}(x, t) & =\chi_{m} u_{m-1}(x, t)+\hbar \mathcal{L}^{-1}\left[R_{1, m}\left(\mathbf{u}_{m-1}, \mathbf{H}_{m-1}\right)\right] \\
H_{m}(x, t) & =\chi_{m} H_{m-1}(x, t)+\hbar \mathcal{L}^{-1}\left[R_{2, m}\left(\mathbf{u}_{m-1}, \mathbf{H}_{m-1}\right)\right] .
\end{aligned}
$$

By using Eqs.(11) and (12) with initial conditions given by (2) we successively obtain

$$
\begin{aligned}
& u_{0}(x, t)=1-\tanh (x / 2), \\
& u_{1}(x, t)=-\frac{\hbar t^{\alpha} e^{x}\left(e^{x}+3\right)}{\Gamma(1+\alpha)\left(e^{x}+1\right)^{3}},
\end{aligned}
$$

and

$$
\begin{aligned}
& H_{0}(x, t)=\frac{3}{4}\left(1-\tanh ^{2}(x / 2)\right), \\
& H_{1}(x, t)=-\frac{3 \hbar t^{\alpha} \operatorname{sech}^{4}(x / 2)(-4+2 \cosh x+\sinh x)}{8 \Gamma(1+\alpha)},
\end{aligned}
$$




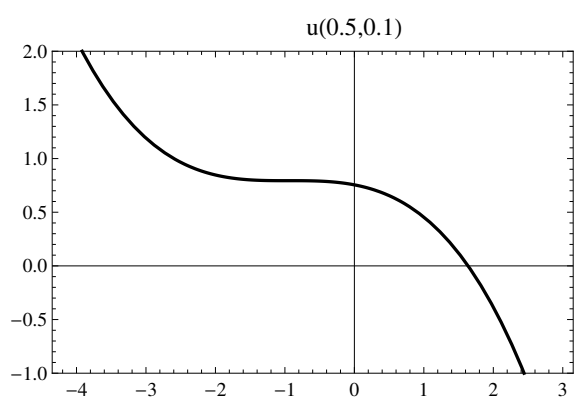

(a) $\mathrm{u}(\mathrm{x}, \mathrm{t})$

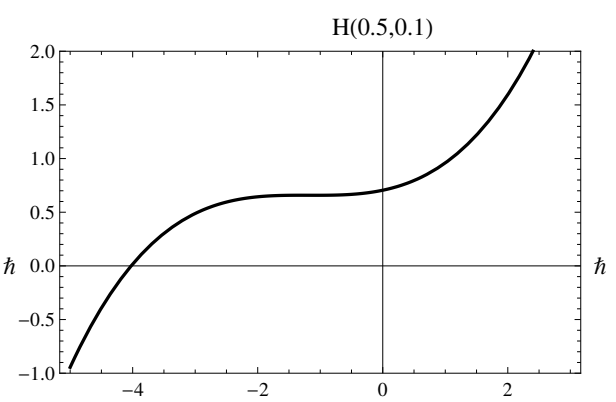

(b) $\mathrm{H}(\mathrm{x}, \mathrm{t})$

Figure 1: The $\hbar$-curves of 3 th-order approximate solutions obtained by the HAM for $\alpha=1$.

Therefore, the series solutions expressed by HAM can be written in the forms

$$
\begin{gathered}
u(x, t)=u_{0}(x, t)+u_{1}(x, t)+u_{2}(x, t)+\ldots \\
H(x, t)=H_{0}(x, t)+H_{1}(x, t)+H_{2}(x, t)+\ldots
\end{gathered}
$$

To demonstrate the efficiency of the method, we compare the HAM solutions of the time fractional Whitham-Broer-Kaup equation given by Eqs. (13) and (14) for $\alpha=1$ with its exact solutions [9]

$$
\begin{aligned}
& u(x, t)=1-\tanh (x / 2-t / 2), \\
& H(x, t)=\frac{3}{4}\left(1-\tanh ^{2}(x / 2-t / 2)\right) .
\end{aligned}
$$

The convergence of the HAM solutions series can be controlled and adjusted simply by the help of the auxiliary parameter $\hbar$ which is in our solution series. To control and adjust the convergence of our solutions series, we search for the suitable range, especially suitable value, for the auxiliary parameter by using so called $\hbar$-curve. We obtain this range and value by discovering the valid region of $\hbar$ which corresponds to the line segments nearly parallel to the horizontal axis, as pointed by Liao [2].

In Fig.1, we demonstrate the $\hbar$-curves of $u(0.5,0.1)$ and $H(0.5,0.1)$ given by 3th-order HAM solutions (13) and (14) for $\alpha=1$. It can be seen from the figures that the valid range of $\hbar$ is approximately $-1.8 \leq \hbar \leq-0.2$. In Fig.2, we demonstrate the $\hbar$-curves of $u(0.5,0.1)$ and $H(0.5,0.1)$ given by 3th-order HAM solutions (13) and (14) for $\alpha=0.75$. It can be seen from the figures 


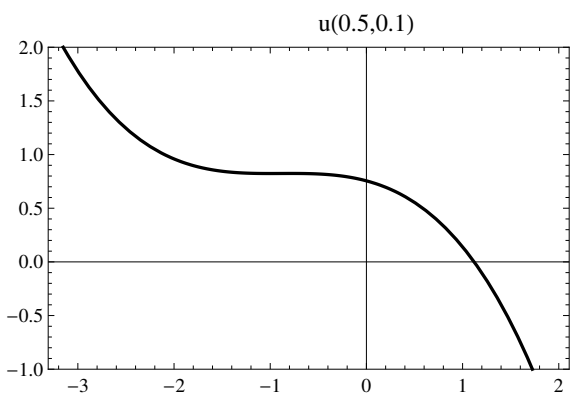

(a) $\mathrm{u}(\mathrm{x}, \mathrm{t})$

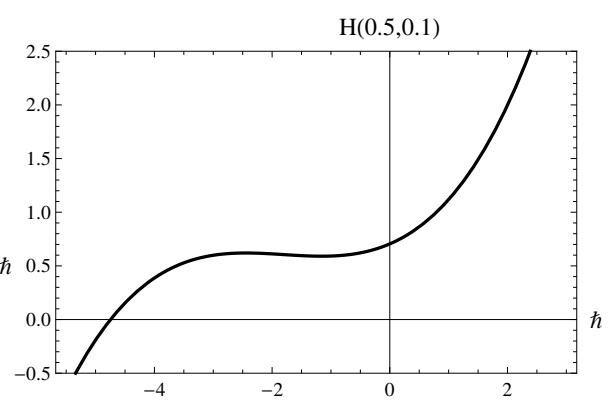

(b) $\mathrm{H}(\mathrm{x}, \mathrm{t})$

Figure 2: The $\hbar$-curves of 3 th-order approximate solutions obtained by the HAM for $\alpha=0.75$.

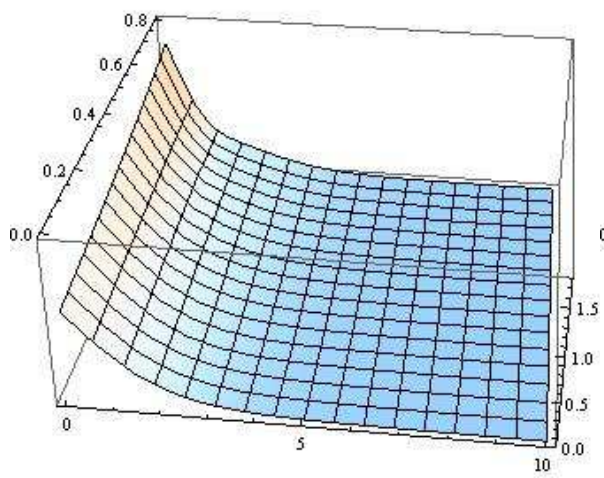

(a) Approximate Solution

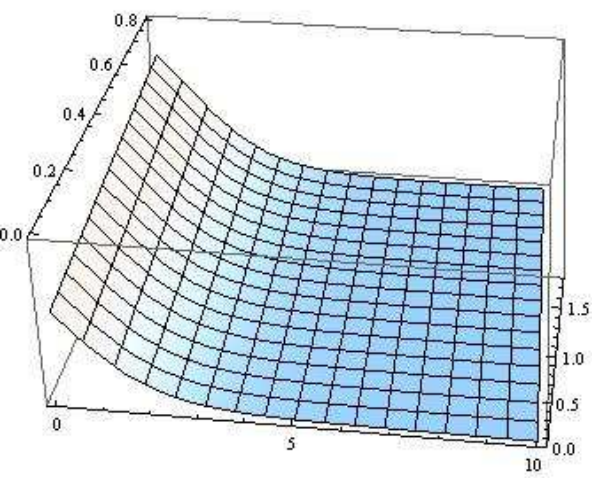

(b) Analytical Solution

Figure 3: The graphs of analytical solution and 3th-order approximate solution obtained by the HAM of $u(x, t)$ for $\alpha=1$ and $\hbar=-0.6$.

that the valid range of $\hbar$ is approximately $-1.4 \leq \hbar \leq-0.2$. Fig. $3-4$ show the numerical solution of $u(x, t)$ and $H(x, t)$ at $\alpha=1$ and $\hbar=-0.6$ obtained by 3 th-order HAM and analytical solution, respectively. Fig. 5 shows the numerical solutions of $u(x, t)$ and $H(x, t)$ at $\alpha=0.75$ and $\hbar=-0.6$ obtained by 3th-order HAM, respectively. 


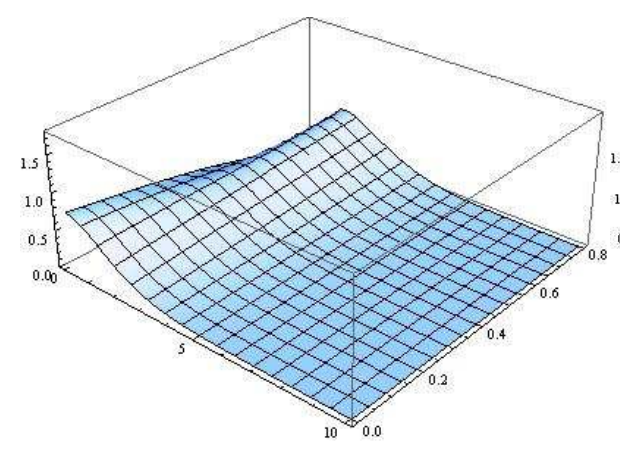

(a) Approximate Solution

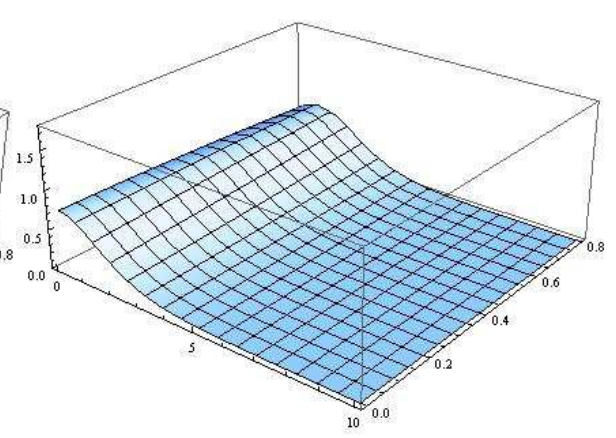

(b) Analytical Solution

Figure 4: The graphs of analytical solution and 3th-order approximate solution obtained by the HAM of $H(x, t)$ for $\alpha=1$ and $\hbar=-0.6$.

\section{Conclusion}

In this paper, the HAM has been successfully applied to obtain approximate analytical solution of the time fractional Whitham-Broer-Kaup equation. It has been also seen that the HAM solution of the problem converges very rapidly to the exact one by choosing an appropriate auxiliary parameter $\hbar$. In conclusion, this study shows that the HAM is a powerful and efficient technique in finding the approximate analytical solution of the time fractional Whitham-Broer-Kaup equation and also many other nonlinear evolution equations arising in science and engineering.

\section{References}

[1] S.J. Liao, The proposed homotopy analysis tecnique for the solution of nonlinear problems, Ph.D thesis, Shanghai Jiao Tong University (1992).

[2] S.J. Liao, Beyond Perturbation: Introduction to the Homotopy Analysis Method, Chapman and Hall/CRC Press, Boca Raton (2003).

[3] S.J. Liao, Homotopy analysis method: A new analytical technique for nonlinear problems, Commun. Nonlinear Sci. Numer. Simulat., 2(2) (1997), $95-100$.

[4] S.J. Liao, On the homotopy analysis method for nonlinear problems, Appl. Math. Comput., 147 (2004), 499-513. 


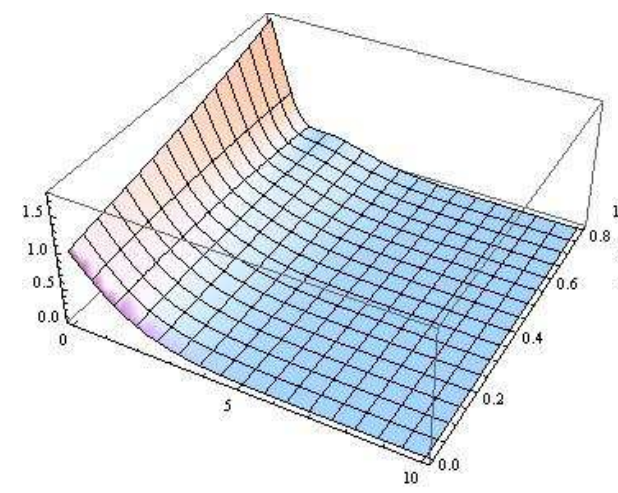

(a) $\mathrm{u}(\mathrm{x}, \mathrm{t})$

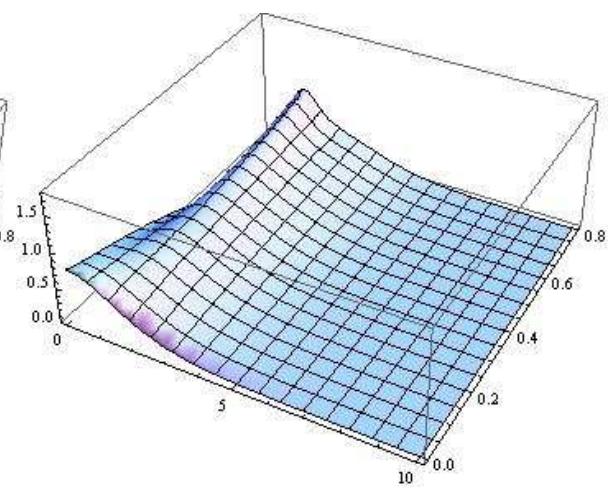

(b) $\mathrm{H}(\mathrm{x}, \mathrm{t})$

Figure 5: The graphs of 3th-order approximate solution obtained by the HAM of $H(x, t)$ for $\alpha=0.75$ and $\hbar=-0.6$.

[5] S.J. Liao, Notes on the homotopy analysis method: Some definitions and theorems, Commun. Nonlinear Sci. Numer. Simulat., 14 (2009), 983-997.

[6] S. Abbasbandy, M.S. Hashemi, I. Hashim, On convergence of homotopy analysis method and its application to fractional integro-differential equations, Quaestiones Mathematicae 36 (2013), 93-105.

[7] S. Abbasbandy, M. Jalili, Determination of optimal convergence-control parameter value in homotopy analysis method, Numerical Algorithms, 64 (2013), 593-605.

[8] A. Esen, N.M. Yagmurlu, O. Tasbozan, Approximate Analytical Solution to Time-Fractional Damped Burger and Cahn-Allen Equations, Applied Mathematics Information Sciences, 7 (5) (2013), 1951-1956.

[9] M.A. Abdou, Further improved F-expansion and new exact solutions for nonlinear evolution equations, Nonlinear Dyn., 52 (2008), 277-288. 